\title{
Vacinas Anticovid: um Olhar da Saúde Coletiva
}

\author{
Anti-Covid vaccines: a look from the Collective Health
}

Reinaldo Guimarães (https://orcid.org/0000-0002-0138-9594) ${ }^{1}$

${ }^{1}$ Núcleo de Bioética e

Ética Aplicada, Universidade

Federal do Rio de Janeiro.

R. Sacopã 191/102,

Lagoa. 22471-180 Rio de

Janeiro RJ Brasil.

reinaldo.guimaraes47@

gmail.com

\begin{abstract}
The paper discusses the complex nature of the pandemic by highlighting the various intrinsic and extrinsic dimensions in the development of SARS-CoV-2 vaccines, with an emphasis on the two most advanced products in clinical testing, namely, the vaccine developed by the University of Oxford associated with the British pharmaceutical company AstraZeneca, and the one developed by Chinese company Sinovac. This choice also stems from the fact that both have testing activities, which, if successful, will lead to future production in Brazil, by Bio-Manguinhos/Fiocruz, Rio de Janeiro, and the Butantã Institute, in São Paulo, respectively. From a conceptual viewpoint, this paper builds on the reflection from the field of Collective Health that addresses the boundaries between the biological and the social spheres. It also seeks to show that, if successful and while important tools for coping with the pandemic, vaccines will not dispense with the continuity of other non-pharmacological measures already used.
\end{abstract}

Key words COVID-19, Collective health, Vaccines
Resumo $O$ artigo discute a complexidade da pandemia destacando as várias dimensões, intrínsecas e extrínsecas envolvidas no desenvolvimento das vacinas contra o SARS-CoV-2, com ênfase nos dois produtos mais avançados no campo dos testes clínicos. São eles, a vacina desenvolvida pela Universidade de Oxford associada à farmacêutica britânica AstraZeneca e a desenvolvida pela empresa chinesa Sinovac. Essa escolha deriva também do fato das duas estarem com atividades de testagem e, caso bem-sucedidas, com futura produção no Brasil, respectivamente pelo Bio-Manguinhos, na Fiocruz, e pelo Instituto Butantã, em São Paulo. Do ponto de vista conceitual, o artigo parte da reflexão oriunda do campo da Saúde Coletiva que trata das fronteiras entre o biológico e o social. Procura ainda demonstrar que, caso sejam bem sucedidas, as vacinas, muito embora importantes ferramentas para o enfrentamento da pandemia, não dispensarão a continuidade de outras medidas não farmacológicas já utilizadas.

Palavras-chave COVID-19, Saúde coletiva, Vacinas 


\section{O objeto da Saúde Coletiva é constituído nos limites do biológico e do social e compreende a investigação dos determinantes da produção social das doenças e da organização dos serviços de saúde... \\ [Jairnilson Paim, 1982] ${ }^{1}$}

\section{A abordagem de temas complexos}

Doenças de massa costumam ser complexas. No caso da pandemia pelo SARS-CoV-2, a complexidade foi agravada, em seu início, pelo desconhecimento quase completo das características do patógeno que a causava e das consequências disso. Na dimensão de sua biologia, as pistas existentes remetiam ao conhecimento de outros coronavírus já identificados que, ao fim e ao cabo, pouco ajudaram no manejo do novo organismo. Em sua fisiopatologia, o que se pensava ser uma enfermidade respiratória revelou-se uma condição sistêmica. No plano da abordagem clínica, mais surpresas com uma evolução heterodoxa na qual sintomas prodrômicos transformavam-se rapidamente em doença grave, sem que o bom estado geral dos pacientes fosse condizente com a gravidade de sua real função respiratória medida pelo oxímetro. No terreno epidemiológico, o acompanhamento do estado imunitário da população também surpreendeu pela pouca presença de portadores de anticorpos quando comparada com a experiência de outras epidemias virais e isso levanta atualmente intenso debate sobre os mecanismos imunológicos envolvidos na doença. No plano dos serviços de saúde, porque a velocidade do adoecimento e a gravidade de parte dos pacientes revelou-se maior e mais intensa do que a organização ordinária dos mesmos estava preparada para suportar. Além dos serviços, a vida em sociedade, no campo do trabalho, do afeto, do lazer, etc., foi também inesperadamente desorganizada, assim como a economia dos países, já bastante fragilizada antes mesmo da pandemia. Apesar de intensa, como veremos a seguir, os resultados da busca por medicamentos eficazes foram frustrantes até o momento. E agora temos uma corrida por uma ou mais boas vacinas.

Uma lição deve ser tirada a partir da descrição feita acima. Não haverá apenas uma medida ou mesmo o ataque a uma das dimensões descritas acima que seja capaz de resolver, per se, o problema em seu conjunto. $\mathrm{O}$ seu enfrentamento deve ser organizado a partir de ações articuladas nas múltiplas dimensões apontadas. O corolário dessa assertiva é que uma ou mais boas vacinas serão importantíssimas para contribuir para en- frentar a COVID 19, mas é muito pouco provável que possam sozinhas resolver o problema em sua totalidade. Por outro lado, para que uma ou mais boas vacinas cumpram seu importante papel, serão necessárias várias etapas e vários aspectos intrínsecos e extrínsecos às mesmas que deverão ser estabelecidos antes que elas possam cumprir a sua missão. Este texto tem o objetivo de discutir a complexidade no campo das vacinas.

\section{A eficácia e a segurança}

Atualmente, (15/7/2020) há no mundo 23 vacinas sendo testadas em seres humanos sendo apenas duas na última etapa dessa testagem (fase 3). Há 140 candidatas em fases anteriores de desenvolvimento ${ }^{2}$. Atualmente o Brasil está envolvido no desenvolvimento clínico das duas. O Instituto Butantã está associado com a empresa chinesa Sinovac e a Fiocruz/Biomanguinhos com a AstraZeneca.

Uma vacina contra o Sars-Cov-2, para ser comercializada e aplicada, ao final dessa fase de testes em humanos, deve demonstrar ser segura, com poucos efeitos colaterais e eficaz. Mas, na situação de emergência em que vive o mundo, algumas delas, como veremos, já estão sendo comercializadas antes de serem estabelecidas as suas segurança e eficácia. Em situações normais, ela deve ter a aprovação da Organização Mundial da Saúde (OMS) e da agência sanitária do país que vai utilizá-la (Anvisa no Brasil). A Organização Mundial da Saúde e a agência sanitária dos Estados Unidos (FDA) decidiram aprovar apenas vacinas capazes de prevenir ou impedir a ocorrência de casos graves em mais de $50 \%$ dos vacinados ${ }^{3}$.

Toda testagem clínica embute alguma probabilidade de insucesso, variável segundo as três passagens de fases (Fase I para II, fase II para III e fase III para a aprovação). De acordo com Lo e $\mathrm{Siah}^{4}$, que analisaram 881 testes clínicos com vacinas candidatas contra agentes infecciosos entre 2000 e 2015, a probabilidade agregada de sucesso para as três passagens de fase foi de $33,4 \%$. Na passagem de interesse para este texto (Fase III para aprovação), que envolveu a análise de 269 ensaios clínicos, a probabilidade de sucesso foi de $85,1 \%( \pm 2,2 \%)$. Esse é, portanto, o cenário que se apresenta no momento para as duas vacinas atualmente envolvidas em ensaios na Fase III.

Os próximos dois critérios a serem discutidos (efetividade e eficiência), caso não sejam satisfatórios, não impedem a priori a comercialização da vacina. Entretanto, são decisivos para a utilização em programas públicos de vacinação em massa. 


\section{A efetividade da vacina}

A eficácia e a segurança são estabelecidas com base na observação entre o produto candidato e um humano em ambiente controlado. Entretanto, vacinas eficazes e seguras devem abraçar critérios que vão além das características intrínsecas do produto. Critérios que medeiam entre as tecnologias que lhes são inerentes e a sua chegada aos organismos das pessoas que pertençam às populações-alvo a que se destinam. São critérios relativos à sua efetividade.

Uma boa vacina deverá fornecer uma memória imunológica longa, se possível deve proteger ao longo de toda uma vida. Se não, por uma ou mais décadas de vida. Ela não deve apresentar manifestações de enhancement, o que significa provocar (ou agravar) a doença que deveria impedir ou atenuar certos subconjuntos de vacinados. Recentemente, uma vacina contra a Dengue (Dengvaxia), aprovada pela OMS e devidamente registrada na Anvisa, além de uma eficácia sofrível, revelou-se perigosa por aumentar a chance da ocorrência de formas graves da doença em pessoas que nunca haviam entrado em contato com o vírus. Por esses motivos, o SUS não a incorporou. Outro exemplo ocorre com as vacinas disponíveis contra a poliomielite. A vacina Sabin, elaborada a partir de vírus vivo atenuado, aumentou a efetividade do combate à doença por ser administrada pela via oral e por permitir que esse vírus atenuado imunizasse outras pessoas quando dispersado pelos vacinados. Mas, contendo um vírus íntegro, foram identificados raros casos de pólio causados pelo vírus vacinal. Em situações onde a incidência da doença se torna muito baixa isso pode se tornar um problema importante, pois o risco de adoecer em vacinados chega perto do risco de adoecer pelo vírus "selvagem”. Daí que alguns sistemas de saúde vêm substituindo a vacina Sabin pela anterior, a vacina Salk, desenvolvida mediante de vírus inativado. No Brasil, o esquema atual prescreve a vacina Salk nas três doses abaixo de um ano de idade e a Sabin nos reforços posteriores ${ }^{5}$.

Até este momento, não há qualquer evidência de enhancement nos testes de vacinas candidatas contra o SARS-CoV-2 em humanos. Em testes pré-clínicos, entretanto, tal fato foi observado no desenvolvimento de vacinas contra outro SARSCoV. O tema é comentado por Hotez et al. ${ }^{6}$

Ainda como desafio à efetividade, uma vacina deve atingir ampla cobertura nas populações-alvo mediante campanha de vacinação adequada (logística, cadeia de frio, etc.). Nesse aspecto, o
Brasil tem uma vantagem importante pela existência do Programa Nacional de Imunizações do SUS (PNI/SUS), portador de larga experiência (46 anos) na dispensação de uma robusta cesta de vacinas no país. A despeito dos desafios logísticos decorrentes da nossa dimensão geográfica, as cerca de 30 mil salas de vacinação habitualmente mobilizadas em campanhas nacionais diminuem problemas dessa ordem.

Será necessário também superar os movimentos ideológicos multinacionais antivacinas. Nascidos no final dos anos 1990 na Europa, a onda atual desse movimento vem crescendo em todo o mundo, em particular nos países do hemisfério norte. Johnson et al. ${ }^{7}$ apresentam dados e discutem a situação atual desses movimentos. Mais especificamente no que se refere a uma vacina contra o Sars-CoV-2, pesquisas de opinião nos Estados Unidos mostram uma situação preocupante. Apenas metade da população norte americana está convicta de tomar uma vacina contra a COVID 19 e $25 \%$ estão indecisos ${ }^{8}$.

No Brasil, muito em função dos bons serviços prestados pelo PNI/SUS, os movimentos antivacinas não prosperaram como aconteceu na América do Norte e na Europa. Entretanto, está vivo. Desde 2019 vem ocorrendo entre nós um surto sustentado de casos de sarampo, com cerca de 15 mil casos naquele ano e cujo epicentro é a cidade de São Paulo e seu entorno. Vários fatores parecem estar contribuindo para o recrudescimento de uma doença que teve sua transmissão interrompida no país em 2000, após a introdução da vacinação dois anos antes ${ }^{9}$. Dentre as várias razões aventadas para esse surto, há consenso de que a diminuição da cobertura vacinal foi decisiva para a sua ocorrência e as razões para isso foram, ao lado do desfinanciamento do SUS após a Emenda Constitucional 95 de 2016, possivelmente, a absorção implícita ou explícita de ideias do movimento mundial antivacinas. O grande impacto da pandemia de COVID 19 no imaginário da população deve atenuar a adesão a essa ideologia. Entretanto, é importante mencionar esse entrave potencial à efetividade das vacinas e se preparar para combate-lo caso se manifeste.

\section{A eficiência da vacina}

O conceito de eficiência gira, habitualmente, em torno de aspectos econômico-financeiros. Para serem eficientes, vacinas devem obter um balanço positivo nos termos da equação custo/ benefício. Nos últimos 20 anos vêm sendo observadas mudanças importantes no mercado 
mundial de vacinas. Essas têm como principais vetores: (1) uma taxa de crescimento do mercado muito acima da taxa correspondente em medicamentos, a despeito de representarem apenas $2 \%$ a3\% deste mercado; (2) Uma fusão empresarial (e "cultural") cada vez maior com grandes farmacêuticas; (3) uma aceleração no desenvolvimento de vacinas mais complexas e mais caras; (4) um aumento da importância epidemiológica de doenças transmissíveis, levando a um aumento da demanda; (5) avanços biotecnológicos no desenvolvimento e na produção de vacinas; (6) vacinas mais lucrativas, com o aparecimento de vacinas blockbuster (influenza, p. ex.); (7) Maior preocupação da OMS, UNICEF e OPAS com esse tema e aparecimento de organizações filantrópicas e coalizões públicas e privadas voltadas às vacinas; (8) Organização pelas empresas de novas estratégias de marketing (pharma-like marketing).

Uma parte dessas mudanças de mercado está relacionada ao que entendo como uma "captura" das empresas produtoras de vacinas pelas grandes farmacêuticas. Entre 2005 e 2012, as 13 maiores compras e fusões entre produtores de vacinas e farmacêuticas movimentaram cerca de US $\$ 220$ bilhões e transformaram, no final desse período, as farmacêuticas GSK, Sanofi, Pfizer, Merck e Novartis nos maiores produtores globais, sendo então responsáveis por cerca de $75 \%$ do mercado global de vacinas ${ }^{10}$. Atualmente, o ranking pode estar modificado, mas o panorama permanece o mesmo. O processo de fusões e aquisições, entre outras consequências, fez com que certas práticas éticas e comerciais da Big Pharma, amplamente conhecidas e muitas vezes condenadas, migrassem para a indústria de vacinas, outrora bem mais comprometidas com a saúde pública. Vacinas tornaram-se Big Business.

O impacto da pandemia medido pelo número de casos e, principalmente, de óbitos fizeram com que desde o seu início, boa parte da comunidade internacional de pesquisadores voltasse seus esforços para enfrentar a COVID 19. Em $14 / 7 / 2020$, havia 76.645 artigos e pré-prints sobre a COVID 19. Em 10/7/2020, havia 4,271 ensaios clínicos registrados em andamento ${ }^{11}$. Mas, além dos esforços da comunidade científica em todo o mundo, a pandemia mobilizou outras três esferas de poder político e financeiro. Governos, organismos multilaterais e grandes organizações filantrópicas. A circunstância de que a pandemia tenha incidido fortemente na China, na União Europeia e nos Estados Unidos fez com que os centros políticos globais passassem a colocar o problema no centro de suas preocupações. Hu- manitarismo, busca de prestígio e de novos negócios parecem ter sido os móveis dessa notável mobilização.

$\mathrm{O}$ interesse da comunidade científica global parece derivar, em primeiro lugar, de um sentimento humanitário frente à pandemia, tendo como componente lateral a busca de prestígio junto aos pares, às agências de fomento e à sociedade. E essa mobilização, em particular no Hemisfério Norte, foi amparada pela alocação de recursos financeiros extraordinários em uma dimensão raramente vista. Como de hábito, a liderança ficou com os Estados Unidos onde esse aporte atingiu a marca de US $\$ 3,6$ bilhões, alocados no National Institutes of Health ${ }^{12}$. Em outros países, como na China e na União Europeia, também foram observados aportes extraordinários. Vale notar entretanto que, a despeito desses aportes, em decorrência da crise econômica global, os orçamentos de Pesquisa e Desenvolvimento nos países centrais deverão sofrer após a pandemia ${ }^{13}$.

No complexo industrial da saúde, o impacto pandêmico gerou um aumento imediato da demanda por alguns segmentos (respiradores e EPIs) e pela perspectiva de novos produtos nos segmentos farmacêutico e de vacinas. Neste último, essa motivação é comprovada pelos já mencionados números de vacinas candidatas e de ensaios clínicos que emergiram. Aqui, consideradas as práticas comerciais da indústria farmacêutica (ultimamente denominada Biofarmacêutica), tudo indica que o sentimento humanitário foi algo acessório. Além da tradicional e conhecida participação de recursos públicos no desenvolvimento das vacinas em suas fases de prova de conceito (Pré-clínica), o impacto da pandemia gerou uma extensão incomum dessa participação. $\mathrm{Na}$ China, na União Europeia e nos Estados Unidos houve a liberação de recursos públicos para apoiar fases mais avançadas (clínicas) de desenvolvimento e a urgência no desenvolvimento de vacinas fez até mesmo com que governos passassem a financiar a construção de instalações fabris em empresas, bem como antecipar compras de produtos ainda inexistentes em sua forma comercializável. Mais ainda, gerou mudanças nos padrões regulatórios em vários países, no sentido de ajustar as regras à urgência no desenvolvimento e na produção de vacinas. Uma tentativa de harmonização inspirada pelas agências norte americana e europeia foi levada a cabo pela International Coalition of $\mathrm{Me}$ dicines Regulatory Authorities (ICMRA) que, no entanto, apresentou resultados muito genéricos ${ }^{14}$, além de não ser de aplicação obrigatória pelas agências regulatórias nacionais. 
Além do impacto político produzido pela pandemia, o componente humanitário do interesse empresarial no desenvolvimento de vacinas decorreu, provavelmente, do protagonismo que a OMS assumiu frente ao tema. A despeito das dificuldades políticas e financeiras pelas quais passa há vários anos, a OMS abraçou a tarefa e o resultado mais importante dessa atuação foi a construção do mecanismo que tornou viável a extensão do aporte de recursos públicos na busca por vacinas. Entretanto, para concretiza-lo, teve que abrir mão da liderança executiva desse mecanismo, o atribuindo a uma organização filantrópica chamada GAVI - Global Alliance for Vaccine and Immunization, criada em 2000 com o propósito de ampliar o acesso a vacinas em países pobres e muito pobres. O GAVI é apoiado por organismos multilaterais (UNICEF, Banco Mundial, OMS) e privadas (Fundação Gates, indústria biofarmacêutica e outros). O mecanismo elaborado pelo GAVI Chama-se COVAX (Global Vaccine Access FacilityCovax Facility), e propõe a constituição de um pool de recursos financeiros para o apressamento no desenvolvimento de vacinas contra o SARS-CoV-2 destinados a todo o mundo. Para os países pobres e muito pobres, trabalha com a possibilidade de subsídios e doações de vacinas. Para os países de renda média alta e ricos propõe um mecanismo de financiamento público (Gavi Advance Market Commitment for COVID-19 Vaccines - Gavi Covax $A M C)$ para que as empresas apressem o desenvolvimento e a produção de vacinas contra o compromisso de garantia de preferência no fornecimento das doses compradas ou doadas aos países pobres e muito pobres. Até 15 de julho de 2020, 75 países de renda média e alta e 90 países pobres ou muito pobres haviam aderido ao mecanismo ${ }^{15}$. A associação entre a OMS e o GAVI tem ainda a virtude de procurar fazer com que os preços das vacinas sejam acessíveis durante a pandemia. Fica em aberto o que acontecerá após o seu final.

Embora haja de modo geral uma apreciação positiva do acordo entre a OMS e o GAVI, algumas vozes estão colocando perguntas que consideram não estarem ainda adequadamente respondidas. Em resumo, essas questões dizem respeito à falta de transparência nas decisões da GAVI-COVAX, à sua falta de experiência na negociação com os países ricos, à extrapolação de seu mandato - originalmente circunscrito a países muito pobres - e à pergunta sobre o motivo de a OMS não ser a formuladora e executora do plano. Um apanhado desses questionamentos pode ser encontrado em recente documento da organização Médicos Sem Fronteiras ${ }^{16}$.
O Brasil está entre os 75 países aderentes ao Covax Facility mediante o acordo que o Ministério da Saúde/Fiocruz/BioManguinhos estabeleceu com a AstraZeneca/Universidade de Oxford, no valor de US\$ 127 milhões para a aquisição de 30,4 milhões de doses de vacina por eles desenvolvida (projetada pela plataforma de um vetor viral), incluída a transferência de sua tecnologia para BioManguinhos e a possível abertura do mercado latino-americano para a vacina produzida aqui. Essa decisão arrojada pretende garantir algum grau de prioridade no fornecimento da mesma à população brasileira no caso de um final feliz para o produto. Igualmente relevante é a inclusão de cláusula de compensação tecnológica na compra da vacina implicando em transferência da tecnologia, somente possível pelas capacidades tecnológica e produtiva já existentes em Biomanguinhos. Mas, a decisão do MS/Fiocruz não é isenta de riscos, haja vista o grau de incerteza sobre o sucesso do produto ao final dos ensaios clínicos.

Outra importante iniciativa doméstica para a produção de uma vacina contra o SARS-CoV-2, igualmente correta, foi patrocinada pelo governo de São Paulo/Instituto Butantã que, em 11 de junho de 2020, anunciou um acordo com a empresa chinesa Sinovac para a participação em ensaio clínico de fase III, já iniciado no Brasil, e futura produção de sua vacina. Ela utiliza uma plataforma de vírus inativado, na qual o Butantã possui larga experiência de desenvolvimento e produção. Pelo informe do instituto, o acordo assinado se refere à participação no ensaio e inclui também cláusula de compensação tecnológica com transferência da tecnologia. Embora não haja informação clara, tudo indica que o acordo Sinovac/Butantã não esteja sendo realizado nos marcos do mecanismo CovaxFacility.

O governo norte americano e a União Europeia comprometeram-se com a compra antecipada de 400 milhões de doses da vacina da AstraZeneca ao custo de US\$1,2 bilhão cada um ${ }^{17,18}$. É provável que outros países tenham também se associado ao mecanismo CovaxFacility ou a outros acordos como o do Butantã com a Sinovac. É possível que tanto na iniciativa do Ministério da Saúde/Fiocruz quanto do Estado de São Paulo/Butantã, caso as respectivas vacinas venham a conseguir registro na Anvisa, possam sofrer dificuldades no fornecimento das mesmas. Será preciso observar qual a capacidade de produção de cada uma das vacinas no Reino Unido e na China, haja vista que os primeiros lotes serão fornecidos pelos produtores e terão que competir 
com outros acordos de fornecimento, nacionais ou locais. Muito provavelmente os dois acordos prevejam cláusulas referentes às datas de entrega, mas as experiências já vividas no curso da pandemia sugerem que nem sempre prazos contratados têm sido respeitados.

\section{Conclusão}

Este texto procurou abordar a complexidade da pandemia dissecando apenas uma das dimensões de seu enfrentamento - a existência de uma ou mais vacinas contra o SARS-CoV-2 aprovadas e postas à disposição da população brasileira, o que poderá colocar o enfrentamento da pandemia em outro patamar. E essa única dimensão, como foi relatado até aqui, também não é isenta de complexidade e de riscos, estando muito afastada de ser considerada uma "bala de prata", como a imprensa, as autoridades e mesmo os pesquisadores têm veiculado. A nossa abordagem enfatiza também esse lugar de observação e análise que, como nos recorda Jairnilson Paim no começo do texto, está nos "limites do biológico e do social". Daí a importância de lançar sobre o problema um olhar da Saúde Coletiva, cuja maior razão de ser é justamente atuar nessas interfaces. 


\section{Referências}

1. Paim JS. Desenvolvimento teórico-conceitual do ensino em saúde coletiva. In: Abrasco. Ensino da saúde pública, medicina preventiva e social no Brasil. Rio de Janeiro: Abrasco; 1982. p. 4-19.

2. World Health Organization (WHO). Draft landscapeof COVID-19 candidate vacines.21 July 2020 Geneva: WHO. [cited 2020 Jan 30]. Available from: https:/www.who.int/publications/m/item/draftlandscape-of-covid-19-candidate-vaccines

3. Food and Drug Administration (FDA). Coronavirus (COVID-19) Update: FDA Takes Actionto Help FacilitateTimelyDevelopmentof Safe, Effective COVID-19 Vaccines. June 30, 2020. [cited 2020 Jan 30]. Available from: https://www.fda.gov/news-events/press-announcements/coronavirus-covid-19-update-fda-takes-action-help-facilitate-timely-development-safe-effective-covid

4. Lo A, Siah K. Estimation of clinical trial success rates and related parameters. Biostatistics 2019; 20(2):273286.

5. Instituto de Tecnologia em Imunobiológicos Bio-Manguinhos. Salk versus Sabin: dois personagens e suas estratégias contra a pólio. [cited 2020 Jan 30]. Available from: https://www.bio.fiocruz.br/index.php /br/noticias/1736-salk-versus-sabin-dois-personagen -e-suas-estrategias-contra-a-polio.

6. Hotez PJ, Corry DB, Bottazzi ME. COVID-19 vaccine design: the Janus face of immune enhancement. $\mathrm{Na}$ ture Reviews Immunology 2020; 20(348):347-348.

7. Johnson NF, Velásquez N, Restrepo NJ, Leahy R, Gabriel N, El Oud S, Zheng M, Manrique P, Wuchty S, Lupu Y. The online competition between pro- and anti-vaccination views. Nature 2020; 582(7811):230233.

8. Cornwall Jun BW. Just $50 \%$ of Americans plan to get a COVID-19 vaccine. Here' show to win over the rest. [cited 2020 Jun 30]. Available from: https://www.sciencemag.org/news/2020/06/just-50-americans-planget-covid-19-vaccine-here-s-how-win-over-rest

9. Prevots DR, Parise MS, Segatto TCV, Siqueira MM, Santos ED, Ganter B, Perreira MCCQ, Domingues CA, Lanzieri T, Silva Júnior JB. Interruption of measles transmission in Brazil, 2000-2001. J Infect Dis 2003; 187(Supl. 1):S111-S120.

10. Kaddar M. Global Vaccine Market Features and Trends. Geneva: WHO. [cited 2020 Jan 30]. Available from: https://www.who.int/influenza_vaccines_plan/resources/session_10_kaddar.pdf?ua $=1$

11. Nature Index. Coronavirus research publishing: The rise and rise of COVID-19 clinical trials. [cited 2020 Jul 16]. Available from: https://www.natureindex. com/news-blog/the-top-coronavirus-research-articles-by-metrics
12. Kaiser J. NIH grapples with rush to claim billions in pandemic research funds. Science. [cited 2020 Jun 3]. Available from: https://www.sciencemag.org/ news/2020/06/nih-grapples-researchers-rush-claimbillions-pandemic-research-funds

13. Sputnik moment or budget breaker: How will the pandemic alter research funding? Nature News Feature. [cited 2020 Jun 2]. Available from: https://www.nature.com/articles/d41586-020-01519-x

14. International Coalition oh Medicines Regulatory Authorities - ICMRA. SARS-CoV-2 Vaccines Workshop \#2 - Summary - July 2020. [cited 2020 Jul 30]. Available from: http://www.icmra.info/drupal/news/ 22june2020/summary

15. World Health Organization (WHO). More than 150 countries engaged in COVID-19 vaccine global access facility. Geneva: WHO; 2020. [cited 2020 Jul 15]. Available from: https://www.who.int/news-room/detail/15-07-2020-more-than-150-countries-engagedin-covid-19-vaccine-global-access-facility

16. Médicos sem Fronteiras (MSF). GAVI Must Ensure COVID-19 vaccines produced through its new global fund for vaccine development are affordable for people everywhere. [cited 2020 Jun 23]. Available from: https://www.doctorswithoutborders.org/what-wedo/news-stories/news/msf-gavi-must-ensure-covid19-vaccines-produced-through-its-new-global

17. Reuters World News. AstraZeneca agrees to supply Europe with 400 million doses of COVID-19 vaccine. [cited 2020 Jun 13]. Available from: https://www. reuters.com/article/us-health-coronavirus-vaccines/ astrazeneca-agrees-to-supply-europe-with-400-million-doses-of-covid-19-vaccine-idUSKBN23K0HW

18. Merelly A. The US just bought 400 million doses of a coronavirus vaccine that may never exist. Quartz. [cited 2020 May 21]. Available from: https:// qz.com/1858682/the-us-just-bought-400-milliondoses-of-a-coronavirus-vaccine-that-may-never-exist/

Artigo apresentado em 20/07/2020

Aprovado em 21/07/2020

Versão final apresentada em 23/07/2020 
